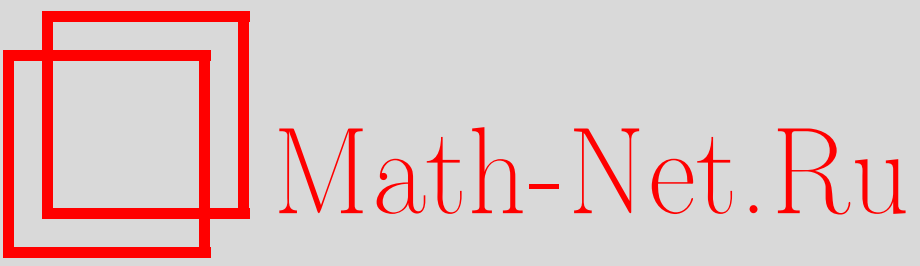

Б. М. Барбашов, В. Н. Первушин, Репараметризационно-инвариантная редукция в гамильтоновом описании релятивистской струны, ТМФ, 2001, том 127, номер 1, 90-109

DOI: https://doi.org/10.4213/tmf450

Использование Общероссийского математического портала Math-Net.Ru подразумевает, что вы прочитали и согласны с пользовательским соглашением

http: //www . mathnet.ru/rus/agreement

Параметры загрузки:

IP : 52.87 .193 .239

26 апреля 2023 г., 12:55:49 
ТЕОРЕТИЧЕСКАЯ

И МАТЕМАТИЧЕСКАЯ

ФИЗИКА

Том 127, № 1

апрель, 2001

(C) 2001 r.

Б. М. Барбашов* , В.Н. Первушин*

\section{РЕПАРАМЕТРИЗАЦИОННО-ИНВАРИАНТНАЯ РЕДУКЦИЯ В ГАМИЛЬТОНОВОМ ОПИСАНИИ РЕЛЯТИВИСТСКОЙ СТРУНЫ}

В рамках обобщенной гамильтоновой теории Дирака путем разрешения связей первого рода изучается времени-репараметризационно-инвариантная динамика релятивистской открытой струны. За репараметризационно-инвариантную эволюционную переменную выбирается временна́я координата "центра масс" струны. Координаты этого "центра масс" отделяются от локальных степеней свободы струны преобразованием, сохраняющим группу диффеоморфизмов обобщенного гамильтониана и пуанкаре-ковариантность локальных связей. Для идентификации временно́й координаты "центра масс" струны с собственным временем, измеряемым в сопутствующей системе координат, используется каноническое преобразование Леви-Чивита-Шанмугадхасана, которое конвертирует глобальную связь (массовую поверхность) в новый импульс так, что для редукции гамильтониана не требуется соответствующего калибровочного условия. Разрешение локальных связей ведет к эквивалентной редуцированной системе с гамильтонианом эволюции относительно собственного времени "центра масс" струны. Квантовая теория такой системы описывается с помощью метода квантования релятивистской струны Рорлиха, в котором участвуют только операторы Вирасоро $L_{n}$ для $n>0$. Все трудности традиционной схемы квантования, включая проблему размерности пространства-времени $D=26$ и существования тахиона, возникают здесь только в случае безмассовой струны $M^{2}=0$.

\section{1. ВВЕДЕНИЕ}

Проблема гамильтоновой формулировки репараметризационно-инвариантных релятивистских систем (частица, струна, модели общей теории относительности (ОТО)) имеет длинную историю [1]-[8]. Такая формулировка основывается на выборе так называемой кинеметрической системы отсчета [9], представляющей собой расслоение пространственно-временного многообразия на множество пространственно-подобных гиперповерхностей, нанизанных на ось времени. Соответствуюшая параметризация метрики была введена независимо Дираком [10], Арновитом, Дезером, Мизнером (АДМ) [11] и Зельмановым [12]. Группа диффеоморфизмов кинеметрической системы отсчета содержит абелеву подгруппу репараметризации координатного времени.

* Объединенный институт ядерных исследований, Дубна, Московская область, Россия 
Изначально этот подход развивался Дираком для обобщенной гамильтоновой формулировки гравитации [6] с целью отделения физического сектора переменных на поверхности связей от “лишних" переменных, ответственных за калибровочный произвол. Такое отделение физического сектора переменных называется редукцией.

Все известные описания релятивистской струны [13], [14] основаны на редуцировании фазового пространства путем наложения калибровочных условий, которые с самого начала нарушают репараметризационную инвариантность теории. В этой связи представляет интерес выяснить, насколько такое описание адекватно репараметризационно-инвариантной “истинной” динамике исходной свободной струны и можно ли описать эту динамику непосредственно в терминах репараметризационно-инвариантных переменных. Рассмотрению этих проблем и посвящена данная работа.

Нами используется метод репараметризационно-инвариантного описания, развитый для теории гравитации в работах [3], [5], [15]. Он основан на редуцировании действия путем разрешения первичных связей так, что одна из переменных исходного расширенного фазового пространства "выпадает" из физического пространства, преврашаясь в эволюционный параметр редуцированной системы, а ее канонически сопряженный импульс становится гамильтонианом системы. Применение такого инвариантного редуцирования расширенного действия в ОТО позволяет формулировать динамику системы в терминах собственного времени, инвариантного относительно репараметризации, с ненулевым гамильтонианом эволюции. В п. 2.3 статьи приводится простой пример такой редукции.

Важным элементом инвариантной редукции является каноническое преобразование Леви-Чивита-Шанмугадхасана (ЛЧШ) [16], [17], которое линеаризует энергетическую связь, преврашая ее в новый импульс (раздел 4). Этот метод применяется для редукции действия релятивистской струны при описании ее динамики с помошью репараметризационно-инвариантного собственного времени.

Раздел 2 посвящен обобщенному гамильтонову формализму релятивистской струны и постановке проблемы. В разделе 3 переменные локальных возбуждений струны отделяются от координат "центра масс". В разделе 4 ЛЧШ-преобразование применяется для разрешения глобальной связи и введения временно́й переменной “центра масс" как собственного времени глобального движения всей системы. В разделе 5 динамика локальных возбуждений струны описывается в терминах этого времени. Там же формулируется квантовая теория такой системы. В разделе 6 строится производяший функционал для функции Грина релятивистской струны.

\section{2. ОБОБШЕННЫЙ ГАМИЛЬТОНОВ ФОРМАЛИЗМ}

2.1. Система со связями. Рассмотрим функцию действия бозонной релятивистской струны в форме [18]

$$
W=-\frac{\gamma}{2} \int d^{2} u \sqrt{-g} g^{\alpha \beta} \partial_{\alpha} x^{\mu} \partial_{\beta} x_{\mu}, \quad u_{\alpha}=\left(u_{0}, u_{1}\right),
$$

где $x_{\mu}-$ координаты струны в пространстве Минковского с метрикой $\left(x_{\mu} x^{\mu}:=x_{0}^{2}-x_{i}^{2}\right)$, 
а $g_{\alpha \beta}$-метрический тензор в двумерном римановом пространстве мировой поверхности струны.

Построение гамильтоновой динамики базируется на АДМ-представлении двумерной метрики в форме [1]

$$
g_{\alpha \beta}=\Omega^{2}\left(\begin{array}{cc}
\lambda_{1}^{2}-\lambda_{2}^{2} & \lambda_{2} \\
\lambda_{2} & -1
\end{array}\right), \quad \sqrt{-g}=\Omega^{2} \lambda_{1}
$$

с инвариантным интервалом

$$
d s^{2}=g_{\alpha \beta} d u^{\alpha} d u^{\beta}=\Omega^{2}\left[\lambda_{1}^{2} d \tau^{2}-\left(d \sigma+\lambda_{2} d \tau\right)^{2}\right], \quad u_{\alpha}=\left(u_{0}=\tau, u_{1}=\sigma\right),
$$

где $\lambda_{1}$ и $\lambda_{2}$ - известные в ОТО функция смешения и вектор сдвига [19], [20], $\Omega$ - конформньй фактор, который после подстановки (3) в (1) не входит в действие

$$
W=-\frac{\gamma}{2} \int_{\tau_{1}}^{\tau_{2}} d \tau \int_{\sigma_{1}(\tau)}^{\sigma_{2}(\tau)} d \sigma\left[\frac{\left(D_{\tau} x\right)^{2}}{\lambda_{1}}-\lambda_{1} x^{\prime 2}\right]
$$

Здесь

$$
D_{\tau} x_{\mu}=\dot{x}_{\mu}-\lambda_{2} x_{\mu}^{\prime} \quad\left(\dot{x}=\partial_{\tau} x, \quad x^{\prime}=\partial_{\sigma} x\right)
$$

- ковариантная производная относительно метрики (3), которая, так же как действие (4), оказывается инвариантной относительно преобразования параметров $\tau$ и $\sigma$ (см. приложение)

$$
\tau \Rightarrow \tilde{\tau}=f_{1}(\tau), \quad \sigma \Rightarrow \tilde{\sigma}=f_{2}(\tau, \sigma)
$$

Такая группа преобразований в гамильтоновом подходе хорошо известна как группа кинеметрических диффеоморфизмов ОТО [9].

Вариация действия (4) по $\lambda_{1}$ и $\lambda_{2}$ ведет к уравнениям

$$
\frac{\delta W}{\delta \lambda_{2}}=\frac{x^{\prime} D_{\tau} x}{\lambda_{1}}=0 \Rightarrow \lambda_{2}=\frac{\dot{x} x^{\prime}}{x^{\prime 2}}, \quad \frac{\delta W}{\delta \lambda_{1}}=\frac{\left(D_{\tau} x\right)^{2}}{\lambda_{1}^{2}}+x^{\prime 2}=0 \Rightarrow \lambda_{1}^{2}=\frac{\left(\dot{x} x^{\prime}\right)^{2}-\dot{x}^{2} x^{\prime 2}}{\left(x^{\prime 2}\right)^{2}}
$$

Из них можно определить величины $\lambda_{1}$ и $\lambda_{2}$, подстановка которых в действие (4) приводит его к стандартной форме Намбу-Гото [21]

$$
W=-\gamma \int_{\tau_{1}}^{\tau_{2}} d \tau \int_{\sigma_{1}(\tau)}^{\sigma_{2}(\tau)} d \sigma \sqrt{\left(\dot{x} x^{\prime}\right)^{2}-\dot{x}^{2} x^{\prime 2}}
$$

Обобшенная гамильтонова форма действия получается из лагранжевой (4) путем преобразования Лежандра [8]

$$
\begin{aligned}
W & =\int_{\tau_{1}}^{\tau_{2}} d \tau \int_{\sigma_{1}(\tau)}^{\sigma_{2}(\tau)} d \sigma\left(-p_{\mu} D_{\tau} x^{\mu}+\lambda_{1} \phi_{1}\right)= \\
& =\int_{\tau_{1}}^{\tau_{2}} d \tau \int_{\sigma_{1}(\tau)}^{\sigma_{2}(\tau)} d \sigma\left(-p_{\mu} \dot{x}^{\mu}+\lambda_{1} \phi_{1}+\lambda_{2} \phi_{2}\right),
\end{aligned}
$$


где

$$
\phi_{1}=\frac{1}{2 \gamma}\left[p_{\mu}^{2}+\left(\gamma x_{\mu}^{\prime}\right)^{2}\right], \quad \phi_{2}=x^{\prime \mu} p_{\mu},
$$

а обобщенный гамильтониан

$$
\mathcal{H}=\lambda_{1} \phi_{1}+\lambda_{2} \phi_{2}
$$

рассматривается как генератор эволюции этой системы относительно временно́го параметра $\tau$. При этом $\lambda_{1}, \lambda_{2}$ рассматриваются как канонические переменные с нулевыми импульсами

$$
P_{\lambda_{1}}=0, \quad P_{\lambda_{2}}=0
$$

и, следовательно, - как первичные связи первого рода [6], [8]. Уравнения для $\lambda_{1}$ и $\lambda_{2}$

$$
\frac{\delta W}{\delta \lambda_{1}}=\phi_{1}=0, \quad \frac{\delta W}{\delta \lambda_{2}}=\phi_{2}=0
$$

известны как вторичные связи первого рода. Гамильтоновы уравнения движения имеют вид

$$
\frac{\delta W}{\delta x^{\mu}}=\dot{p}_{\mu}-\partial_{\sigma}\left[\gamma \lambda_{1} x_{\mu}^{\prime}+\lambda_{2} p_{\mu}\right]=0, \quad \frac{\delta W}{\delta p^{\mu}}=p_{\mu}-\gamma \frac{D_{\tau} x_{\mu}}{\lambda_{1}}=0 .
$$

Таким образом, проблема состоит в нахождении таких решений уравнений (13) и связей (12), которые инвариантны относительно кинеметрических преобразований (6).

2.2. Метод фиксирования калибровки. Стандартным методом решения такой системы со связями является фиксирование связей второго рода (условий калибровки), которые добавляются к связям первого рода (11) и (12). Например, в теории релятивистской струны выбираются следуюшие связи (ортонормальная калибровка):

$$
\chi_{1}^{(1)}=\lambda_{1}=1, \quad \chi_{2}^{(1)}=\lambda_{2}=0,
$$

которые, конечно, сразу нарушают кинеметрическую симметрию (6) (см. приложение).

В этом случае классические уравнения движения (13) сводятся к линейным уравнениям Даламбера для координат струны $x_{\mu}$,

$$
\partial_{\tau}^{2} x_{\mu}-\partial_{\sigma}^{2} x_{\mu}=0
$$

дополненным определенными граничными условиями. Выбирая случай свободной открытой струны, для которой граничными условиями являются условия $x^{\prime}\left(\tau, \sigma_{1,2}\right)=0$, $\sigma_{1}=0, \sigma_{2}=\pi$, для решения уравнения (15) имеем [14]

$$
x_{\mu}(\tau, \sigma)=X_{\mu}(\tau)+\xi_{\mu}(\tau, \sigma)=Q_{\mu}+\frac{P_{\mu}}{\pi \gamma} \tau+\frac{1}{2 \sqrt{\pi \gamma}}\left[\psi_{\mu}(\tau+\sigma)+\psi_{\mu}(\tau-\sigma)\right],
$$

где $\psi_{\mu}$ - периодические функции с периодом $2 \pi$, представимые рядами Фурье

$$
\psi_{\mu}(z)=i \sum_{n \neq 0} \exp (-i n z) \frac{\alpha_{n \mu}}{n}, \quad \alpha_{-n, \mu}=\alpha_{n, \mu}^{*} .
$$


Величина $X_{\mu}(\tau)=Q_{\mu}+\tau P_{\mu} / \pi \gamma$ задает координату "центра масс" струны, определяя ее равномерное движение как целого:

$$
X_{\mu}(\tau)=\frac{1}{\pi} \int_{0}^{\pi} d \sigma x_{\mu}(\tau, \sigma) .
$$

Переменные $\xi_{\mu}$ являются координатами локальных возбуждений струны, не содержаших нулевых фурье-гармоник,

$$
\int_{0}^{\pi} d \sigma \xi_{\mu}(\tau, \sigma)=\frac{1}{2 \sqrt{\pi \gamma}} \int_{0}^{\pi} d \sigma\left[\psi_{\mu}(\tau+\sigma)+\psi_{\mu}(\tau-\sigma)\right]=0
$$

Полньй импульс струны $P_{\mu}$ и функция $\psi_{\mu}$ подчиняются вторичным связям первого рода (12),

$$
\phi_{1} \pm \phi_{2}=0 \Rightarrow\left[\underline{P}_{\mu}+\psi_{\mu}^{\prime}(\tau \pm \sigma)\right]^{2}=0, \quad \underline{P}_{\mu}=\frac{P_{\mu}}{\sqrt{\pi \gamma}}=\alpha_{0 \mu} .
$$

Эти связи, записанные через ряды Фурье (17),

$$
\phi_{1} \pm \phi_{2}=-\frac{1}{\pi} \sum_{n=-\infty}^{+\infty} L_{n} \exp \left(-i z_{ \pm} n\right)=0 \quad\left(z_{ \pm}=\tau \pm \sigma\right)
$$

приводят к условиям на коэффициенты $L_{n}$,

$$
L_{0}=-\frac{\underline{P}^{2}}{2}+\bar{L}_{0}=0, \quad L_{n \neq 0}=-\underline{P}_{\mu} \alpha_{n}^{\mu}+\bar{L}_{n}=0,
$$

в которых величины $\bar{L}_{n}$ не содержат вкладов от нулевых гармоник $\alpha_{0 \mu}=\underline{P}_{\mu}$,

$$
\bar{L}_{0}=-\frac{1}{2} \sum_{k \neq 0} \alpha_{k \mu} \alpha_{-k}^{\mu}, \quad \bar{L}_{n \neq 0}=-\frac{1}{2} \sum_{k \neq 0, n} \alpha_{k \mu} \alpha_{n-k}^{\mu} .
$$

Как видно из первого равенства $(22), \bar{L}_{0}$ определяет массу струны $\left(P_{\mu}^{2}=M^{2}\right)$

$$
M^{2}=-2 \pi \gamma \bar{L}_{0}=-\frac{\gamma}{2} \int_{0}^{\pi} d \sigma\left[\left(\psi_{\mu}^{\prime}\left(z_{+}\right)\right)^{2}+\left(\psi_{\mu}^{\prime}\left(z_{-}\right)\right)^{2}\right] .
$$

В квантовой теории фурье-коэффициенты $L_{n}(22)$ образуют алгебру Вирасоро с конформной аномалией [13], [14].

Вторичные связи первого рода (20), как было упомянуто выше, при стандартном подходе дополняются калибровочными условиями (первичные связи второго рода), например, в форме [7], [14]

$$
\chi_{1}^{(2)}=n_{\mu} \xi^{\mu}=0, \quad \chi_{2}^{(2)}=n_{\mu} \pi^{\mu}=0,
$$

где $n_{\mu}$-произвольный времениподобный вектор (алгебра связей $\chi_{1,2}^{(2)}, \phi_{1,2}$ дана, например, в работе [7]). 
Таким образом, в приведенном выше стандартном подходе к динамике релятивистской струны обнаруживается, во-первых, что решение (16) выражается через репараметризационно-неинвариантное координатное время $\tau$, которому соответствует благодаря (12) нулевой гамильтониан (10), и, во-вторых, что связи (20) смешивают глобальное движение (полный импульс струны $P_{\mu}$ ) с локальными координатами возбуждений струны $\xi_{\mu}$. Это противоречит релятивистской инвариантности внутренних степеней свободы [22].

В этой связи естественно возникают некоторые вопросы. Возможно ли введение репараметризационно-инвариантной временно́й переменной вместо неинвариантного параметра $\tau$ ? Возможно ли построить ненулевой гамильтониан, описывающий эволюцию координаты "центра масс" струны и какова связь эволюции “центра масс" с унитарным представлением группы Пуанкаре [22]?

2.3. Простой пример инвариантной редукции гамильтониана. Для прояснения ответов на поставленные выше вопросы рассмотрим простой пример времени-репараметризационно-инвариантной классической системы, для которой инвариантная гамильтонова редукция осушествляется путем прямого разрешения связей [3],

$$
W=\int_{\tau_{1}}^{\tau_{2}} d \tau\left(p \dot{q}-\Pi_{0} \dot{Q}_{0}-\lambda\left[-\Pi_{0}+H(p, q)\right]\right),
$$

где $-\Pi_{0}+H(p, q)$ является расширенным гамильтонианом, а действие (26) инвариантно относительно репараметризации временно́го параметра $\tau$,

$$
\tau \rightarrow \tau^{\prime}=\tau^{\prime}(\tau), \quad \lambda \rightarrow \lambda^{\prime}=\lambda \frac{d \tau}{d \tau^{\prime}}
$$

Инвариантная гамильтонова редукция этой расширенной системы означает разрешение следующих трех уравнений, полученных вариацией (26) относительно “лишших” степеней свободы:

$$
\begin{aligned}
& \frac{\delta W}{\delta \lambda}=-\Pi_{0}+H(p, q)=0, \\
& \frac{\delta W}{\delta \Pi_{0}}=0 \Rightarrow \frac{d Q_{0}}{d \tau}=\lambda, \\
& \frac{\delta W}{\delta Q_{0}}=\frac{d \Pi_{0}}{d \tau}=0 .
\end{aligned}
$$

Мы называем эти три уравнения геометрическим сектором расширенного фазового пространства. Из уравнения (28) следует, что $\Pi_{0}=H(p, q)>0$, уравнение (29) определяет $Q_{0}=\int^{\tau} \lambda\left(\tau^{\prime}\right) d \tau^{\prime}$ как репараметризационно-инвариантную эволюционную переменную, которую отождествляют с наблюдаемым (собственным) временем $Q_{0}=t$. Уравнение (30) дает закон сохранения.

Подстановка этих решений в исходное действие (26) редуцирует его к обычной форме

$$
W=\int_{t\left(\tau_{1}\right)=t_{1}}^{t\left(\tau_{2}\right)=t_{2}} d t\left(p \frac{d q}{d t}-H(p, q)\right)
$$


в терминах собственного времени $t$ и ненулевого гамильтониана $H(p, q)$. Таким образом, действие (31) на связях (28)-(30) есть действие в редуцированном фазовом пространстве переменных $(p, q)$ с временем $t$ и ненулевым гамильтонианом. Это действие отличается от полученного с помощью фиксации калибровки $\lambda=1, \tau=t$ прежде всего определением времени, а также ненулевым гамильтонианом эволюции

$$
\lambda\left[-\Pi_{0}+H(p, q)\right] \neq H(p, q) .
$$

Нетрудно убедиться, что уравнения (31) совпадают с инвариантным сектором уравнений исходной системы (26). Далее мы применим метод инвариантной гамильтоновой редукции к релятивистской струне, проверяя на каждом шагу эквивалентность редуцированной системы уравнениям исходной системы и отделяя координаты “центра масс".

\section{3. ОТДЕЛЕНИЕ КООРДИНАТ “ЦЕНТРА МАСС” СТРУНЫ}

Любая протяженная система допускает отделение координат ее центра масс. Для введения репараметризационно-инвариантного времени в теории релятивистской струны по аналогии с введенным в предыдущем разделе временем $t=Q_{0}$ и нахождения переменной, уравнение для которой идентично уравнению (29), рассмотрим координаты центра масс струны, определенные согласно (18) в обшем случае (без наложения калибровки (14)) следуюшим образом:

$$
X_{\mu}(\tau)=\frac{1}{l(\tau)} \int_{\sigma_{1}(\tau)}^{\sigma_{2}(\tau)} d \sigma x_{\mu}(\tau, \sigma), \quad l(\tau)=\sigma_{2}(\tau)-\sigma_{1}(\tau) .
$$

Как будет показано в дальнейшем (см. ниже формулы $(55),(59))$, именно временна́я координата (33) и будет этим временем. Чтобы выразить уравнения движения в терминах этого времени, нам необходимо отделить координаты “центра масс" струны от локальных координат $\xi_{\mu}(\tau, \sigma)$ в действии (4). После подстановки в (4) выражения

$$
x_{\mu}(\tau, \sigma)=X_{\mu}(\tau)+\xi_{\mu}(\tau, \sigma)
$$

оно принимает вид

$$
W=-\frac{\gamma}{2} \int_{\tau_{1}}^{\tau_{2}} d \tau\left\{\frac{\dot{X}^{2} l(\tau)}{N_{0}(\tau)}+2 \dot{X}_{\mu} \int_{\sigma_{1}(\tau)}^{\sigma_{2}(\tau)} d \sigma \frac{D_{\tau} \xi^{\mu}}{\lambda_{1}}+\int_{\sigma_{1}(\tau)}^{\sigma_{2}(\tau)} d \sigma\left(\frac{\left(D_{\tau} \xi\right)^{2}}{\lambda_{1}}-\lambda_{1} \xi^{\prime 2}\right)\right\},
$$

где глобальная функция смещения $N_{0}(\tau)$ определена как функционал от $\lambda_{1}(\tau, \sigma)$ выражением

$$
\frac{1}{N_{0}\left[\lambda_{1}\right]}=\frac{1}{l(\tau)} \int_{\sigma_{1}(\tau)}^{\sigma_{2}(\tau)} d \sigma \frac{1}{\lambda_{1}(\tau, \sigma)} .
$$

Из определения координаты центра масс (33) и равенства (34) следует, что локальные координаты удовлетворяют условию

$$
\int_{\sigma_{1}(\tau)}^{\sigma_{2}(\tau)} d \sigma \xi_{\mu}=0
$$


(см. (19)). Построение канонического формализма предполагает такое же отделение импульса центра масс из сопряженных импульсов $p_{\mu}$, определяемых уравнениями (13). Если подставить в эти уравнения выражение (34), получим

$$
p_{\mu}(\tau, \sigma)=\gamma\left(\frac{\dot{X}_{\mu}(\tau)}{\lambda_{1}}+\frac{D_{\tau} \xi_{\mu}(\tau, \sigma)}{\lambda_{1}}\right)
$$

Определяя полный импульс струны $P_{\mu}$, как и координату (33),

$$
P_{\mu}=\int_{\sigma_{1}(\tau)}^{\sigma_{2}(\tau)} d \sigma p_{\mu}(\tau, \sigma)=\gamma \int_{\sigma_{1}(\tau)}^{\sigma_{2}(\tau)} d \sigma\left(\frac{\dot{X}_{\mu}(\tau)}{\lambda_{1}}+\frac{D_{\tau} \xi_{\mu}(\tau, \sigma)}{\lambda_{1}}\right)
$$

с учетом (36) найдем

$$
P_{\mu}=\gamma \frac{\dot{X}_{\mu} l}{N_{0}(\tau)}+\gamma \int_{\sigma_{1}(\tau)}^{\sigma_{2}(\tau)} d \sigma \frac{D_{\tau} \xi_{\mu}(\tau, \sigma)}{\lambda_{1}}
$$

следовательно, должно выполняться равенство

$$
\int_{\sigma_{1}(\tau)}^{\sigma_{2}(\tau)} d \sigma \frac{D_{\tau} \xi^{\mu}}{\lambda_{1}}=\int_{\sigma_{1}(\tau)}^{\sigma_{2}(\tau)} d \sigma \pi_{\mu}(\tau, \sigma)=0
$$

Это разделение переменных на уровне действия (35) сохраняет группу диффеоморфизмов обобшенного гамильтониана и ведет к обобшенному действию типа Дирака-Бергмана, где переменные центра масс $X_{\mu}, P_{\mu}$ отделены от локальных степеней свободы $\xi^{\mu}$ и $\pi_{\mu}$, которые формируют массу и спин струны. Это действие имеет вид

$$
W=\int_{\tau_{1}}^{\tau_{2}} d \tau\left[\left(\int_{\sigma_{1}(\tau)}^{\sigma_{2}(\tau)} d \sigma\left[-\pi_{\mu} D_{\tau} \xi^{\mu}-\lambda_{1} \mathcal{H}\right]\right)-P_{\mu} \dot{X}^{\mu}+N_{0} \frac{P_{\mu}^{2}}{2 \bar{\gamma}}\right], \quad \bar{\gamma}=\gamma l(\tau)
$$

где $\mathcal{H}$ - гамильтониан локальных возбуждений струны

$$
\mathcal{H}=-\frac{1}{2 \gamma}\left[\pi_{\mu}^{2}+\left(\gamma \xi_{\mu}^{\prime}\right)^{2}\right] \text {. }
$$

Вариация действия (42) по $\lambda_{1}$ приводит к уравнению

$$
\frac{\delta W}{\delta \lambda_{1}}=\mathcal{H}-\left(\frac{1}{l(\tau) \bar{\lambda}(\tau, \sigma)}\right)^{2} \frac{P_{\mu}^{2}}{2 \gamma}=0 .
$$

При выводе этого уравнения из выражения (36) вычисляется вариация функционала

$$
\frac{\delta N_{0}\left[\tau \mid \lambda_{1}\right]}{\delta \lambda_{1}}=\frac{\delta}{\delta \lambda_{1}}\left[\frac{l(\tau)}{\int_{\sigma_{1}(\tau)}^{\sigma_{2}(\tau)} d \sigma \frac{1}{\lambda_{1}(\tau, \sigma)}}\right]=\frac{l(\tau)}{\left[\lambda_{1}(\tau, \sigma) \int_{\sigma_{1}(\tau)}^{\sigma_{2}(\tau)} d \sigma \frac{1}{\lambda_{1}(\tau, \sigma)}\right]^{2}}=\frac{N_{0}^{2}}{l \lambda_{1}^{2}}
$$

4 Теоретическая и математическая физика, т. 127, № 1, 2001 г. 
и вводится инвариантная относительно репараметризации $\tau \rightarrow f(\tau)$ функция смешения

$$
\bar{\lambda}_{1}(\tau, \sigma)=\frac{\lambda_{1}(\tau, \sigma)}{N_{0}(\tau)}
$$

В соответствии с нашим разделением динамических переменных на глобальные и локальные необходимо спроектировать связь первого рода (44) на глобальньй сектор (нулевые фурье-гармоники) и локальный сектор. Глобальная часть связи может быть получена из (44) либо ее умножением на $\bar{\lambda}_{1}(\tau, \sigma)$ и последуюшим интегрированием по $\sigma$, либо (что дает тот же результат) вариацией действия (42) по глобальной функции смещения $N_{0}$. В результате получаем связь

$$
\frac{\delta W}{\delta N_{0}}=\frac{P_{\mu}^{2}}{2 \bar{\gamma}}-H=0, \quad H=\int_{\sigma_{1}}^{\sigma_{2}} d \sigma \bar{\lambda}_{1} \mathcal{H}
$$

которая выражает массу струны $P_{\mu}^{2}=M^{2}$ через гамильтониан локальных степеней свободы $H$. Локальная часть связи (44) получается подстановкой $P_{\mu}^{2}$ из (46) в (44),

$$
\bar{\lambda}_{1} \mathcal{H}-\frac{1}{l \bar{\lambda}_{1}} \int_{\sigma_{1}}^{\sigma_{2}} d \sigma \bar{\lambda}_{1} \mathcal{H}=0
$$

Интегрирование (47) по $\sigma$ приводит к тождеству, если учесть определения $N_{0}$ в $(36)$ и

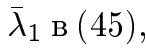

$$
\frac{1}{l(\tau)} \int_{\sigma_{1}(\tau)}^{\sigma_{2}(\tau)} d \sigma \frac{1}{\bar{\lambda}_{1}}=\frac{1}{l(\tau)} \int_{\sigma_{1}(\tau)}^{\sigma_{2}(\tau)} d \sigma \frac{N_{0}(\tau)}{\bar{\lambda}_{1}(\tau, \sigma)}=1
$$

Таким образом, локальная часть связи (47) определена в классе функций ненулевых фурье-гармоник, как и локальные величины в (37) и (41).

Теперь мы можем представить действие (42) с учетом соотношения (47) в эквивалентной форме

$$
W=\int_{\tau_{1}}^{\tau_{2}} d \tau\left[-\int_{\sigma_{1}(\tau)}^{\sigma_{2}(\tau)} d \sigma \pi_{\mu} D_{\tau} \xi^{\mu}-P_{\mu} \dot{X}^{\mu}+N_{0}\left(\frac{P_{\mu}^{2}}{2 \bar{\gamma}}-H\right)\right]
$$

где глобальная функция смешения $N_{0}$ и локальная $\bar{\lambda}_{1}$ рассматриваются как независимые вариационные переменные с учетом нормировки (48) после выполнения вариации.

Из (49) следует, что динамика локальных степеней свободы струны $\pi_{\mu}$ и $\xi^{\mu}$, которые принадлежат классу функций (37) и (41), описьвается уравнениями (13), в которых $x_{\mu}$, $p_{\mu}$ надо заменить на $\xi_{\mu}, \pi_{\mu}$, и связями первого рода (первичными и вторичными)

$$
\pi_{\mu} \xi^{\prime \mu}=0, \quad \bar{\lambda}_{1} \mathcal{H}-\frac{1}{l \bar{\lambda}_{1}} \int_{\sigma_{1}}^{\sigma_{2}} d \sigma \bar{\lambda}_{1} \mathcal{H}=0
$$

Результатом отделения переменных "центра масс" в (49) явилось то, что связи (50) не содержат полньй момент струны $P_{\mu}$ в противоположность стандартным связям (20). 
Поэтому возникает вопрос: когда необходимо отделять координаты "центра масс" струны - до вариации функции действия, как сделано здесь, или после, в уравнениях движения? Релятивистская инвариантность диктует первый способ, поскольку наблюдатель, находящийся в системе "центра масс", не может измерять полньй импульс струны.

Далее, локальные связи первого рода (50) могут быть дополнены связями второго рода (25)

$$
\bar{\lambda}_{1}=1, \quad \lambda_{2}=0, \quad n_{\mu} \xi^{\mu}=0, \quad n_{\mu} \pi^{\mu}=0,
$$

где $n_{\mu}$ - времениподобный вектор. Если выбрать в качестве такого вектора полный импульс струны $P_{\mu}$, то получим с учетом глобальной связи (50) калибровку Рорлиха [23], которая обсуждается в рамках нашего подхода в п. 5.3. После подстановки первых двух связей (51) функция действия (49) становится функцией действия на поверхности локальных связей

$$
W=\int_{\tau_{1}}^{\tau_{2}} d \tau\left[\int_{\sigma_{1}(\tau)}^{\sigma_{2}(\tau)} d \sigma \pi_{\mu} \dot{\xi}^{\mu}-P_{\mu} \dot{X}^{\mu}+N_{0}\left(\frac{P_{\mu}^{2}}{2 \bar{\gamma}}-H\right)\right] .
$$

Отсюда следуют уравнения движения исходной системы для локальных переменных $\pi_{\mu}$, $\xi_{\mu}$, а также глобальная связь (46), разрешая которую относительно $P_{0}$, получаем

$$
\frac{\delta W}{\delta N_{0}}=0 \Rightarrow P_{0}= \pm \sqrt{P_{i}^{2}+2 \bar{\gamma} H} .
$$

Вариация действия $(52)$ по $P_{0}$ и $X_{0}$ дает уравнения

$$
\frac{\delta W}{\delta P_{0}}=-\frac{d X_{0}}{d \tau}+N_{0} \frac{P_{0}}{\bar{\gamma}}=0, \quad \frac{\delta W}{\delta X_{0}}=0 \Rightarrow \frac{d P_{0}}{d \tau}=0,
$$

приводяшие к инвариантному относительно репараметризации $\tau$ определению $X_{0}$ как времени "центра масс" струны, измеряемому наблюдателем в покояшейся системе координат,

$$
d X_{0}=N_{0}(\tau) \frac{P_{0}}{\bar{\gamma}} d \tau
$$

Теперь из (52) с учетом этих равенств мы получаем редуцированное действие на поверхности глобальных (46) и локальных (50) связей

$$
W_{ \pm}=\int_{X_{0}\left(\tau_{1}\right)}^{X_{0}\left(\tau_{2}\right)} d X_{0}\left[\int_{\sigma_{1}\left(X_{0}\right)}^{\sigma_{2}\left(X_{0}\right)} d \sigma \pi_{\mu} \frac{d \xi^{\mu}}{d X_{0}}+P_{i} \frac{d X_{i}}{d X_{0}} \mp \sqrt{P_{i}^{2}+2 \bar{\gamma} H}\right]
$$

записанное в терминах измеряемого в покояшейся системе отсчета времени $X_{0}$ и с нулевым гамильтонианом эволюции. Однако действие (54) ведет к нелинейным уравнениям движения для локальных переменных $\pi_{\mu}, \xi_{\mu}$. Для преодоления этой трудности мы прибегнем к каноническому преобразованию, которое, в частности, дает описание системы в терминах собственного времени для координаты "центра масс" струны. 


\section{4. КАНОНИЧЕСКОЕ ПРЕОБРАЗОВАНИЕ ЛЕВИ-ЧИВИТА}

Преобразование $\left(P_{\mu}, X_{\mu}\right) \Rightarrow\left(\Pi_{\mu}, Q_{\mu}\right)[16],[17],[24]$, определяемое формулами

$$
\Pi_{0}=\frac{1}{2 \bar{\gamma}}\left(P_{0}^{2}-P_{i}^{2}\right), \quad \Pi_{i}=P_{i}, \quad Q_{0}=X_{0} \frac{\bar{\gamma}}{P_{0}}, \quad Q_{i}=X_{i}-X_{0} \frac{P_{i}}{P_{0}},
$$

позволяет превратить глобальную связь (46) в новый импульс $\Pi_{0}=H$, а временну́ю компоненту "центра масс" $X_{0}$, заданную равенством (53), перевести во временну́ю координату $d Q_{0}=N_{0}(\tau) d \tau$, которую будем называть собственным временем "центра масс". Из формул (55) "старые" импульсы $P_{\mu}$ и координаты $X_{\mu}$ выражаются через "новые" следуюшим образом:

$$
\begin{aligned}
P_{0}= \pm \sqrt{2 \bar{\gamma} \Pi_{0}+\Pi_{i}^{2}}, & P_{i}=\Pi_{i}, \\
X_{0}= \pm Q_{0} \frac{\sqrt{2 \bar{\gamma} \Pi_{0}+\Pi_{i}^{2}}}{\bar{\gamma}}, & X_{i}=Q_{i}+Q_{0} \frac{\Pi_{i}}{\bar{\gamma}}
\end{aligned}
$$

что дает возможность записать расширенное действие (49) в новых переменных (ср. (26))

$$
W=\int_{\tau_{1}}^{\tau_{2}} d \tau\left[-\int_{\sigma_{1}(\tau)}^{\sigma_{2}(\tau)} d \sigma \pi_{\mu} D_{\tau} \xi^{\mu}-\Pi_{\mu} \dot{Q}^{\mu}+N_{0}\left(\Pi_{0}-H\right)-\frac{d}{d \tau}\left(Q_{0} \Pi_{0}\right)\right] .
$$

Для глобальных переменных $Q_{0}, \Pi_{0}$ редукция гамильтониана означает, как и в простом примере п. 2.3, разрешение связи (46) относительно компоненты импульса П 0

$$
\frac{\delta W}{\delta N_{0}}=0 \Rightarrow \Pi_{0}=H,
$$

а варьирование действия по П 0

$$
\frac{\delta W}{\delta \Pi_{0}}=0 \Rightarrow \frac{d Q_{0}}{d \tau}=N_{0}(\tau)
$$

определяет новую переменную $Q_{0}$ как собственное время "центра масс" струны $t$ с точностью до размерного фактора

$$
\sqrt{\gamma} t=Q_{0}=\int^{\tau} d \bar{\tau} N_{0}(\bar{\tau}) .
$$

Уравнение для $Q_{0}$, как и в $(32)$, приводит к закону сохранения

$$
\frac{\delta W}{\delta Q_{0}}=0 \Rightarrow \frac{d \Pi_{0}}{d \tau}=0, \quad \text { т.е. } \frac{d H}{d t}=0 .
$$

Таким образом, из (57) получаем аналогично (27) редуцированное действие для струны в терминах собственного времени $t$, глобальных переменных $Q_{i}, \Pi_{i}$ и локальных переменных $\xi_{i}, \pi_{i}$,

$$
W^{\mathrm{R}}=\int_{t_{1}}^{t_{2}} d t\left[-\int_{\sigma_{1}(t)}^{\sigma_{2}(t)} d \sigma \pi_{\mu} D_{t} \xi^{\mu}+\Pi_{i} \frac{d Q_{i}}{d t}-H-\frac{d}{d t}(t H)\right],
$$


где по аналогии с (45) введен факторизованньй “вектор смещения" $\lambda_{2}=N_{0}(\tau) \bar{\lambda}_{2}(\tau, \sigma)$, а ковариантная производная (5) при переходе от $\tau$ к $t$ определена равенством

$$
D_{t} \xi^{\mu}=\partial_{t} \xi^{\mu}-\bar{\lambda}_{2} \xi^{\prime \mu}=\frac{D_{\tau} \xi^{\mu}}{N_{0}}
$$

Редуцированная система (61) имеет тривиальное решение для глобальных переменных $Q_{i}, \Pi_{i}$

$$
\frac{\delta W^{\mathrm{R}}}{\delta Q_{i}}=0 \Rightarrow \frac{d \Pi_{i}}{d t}=0, \quad \frac{\delta W^{\mathrm{R}}}{\delta \Pi_{i}}=0 \Rightarrow \frac{d Q_{i}}{d t}=0,
$$

которые теперь согласно (55) должны рассматриваться как начальные данные для $X_{i}$ и $P_{i}$, поскольку, подставляя решения (58), (59) и (63) в (56) с учетом глобальной связи (46), имеем

$$
P_{0}= \pm \sqrt{M^{2}+P_{i}^{2}}, \quad X_{0}(t)=\frac{P_{0}}{\sqrt{\gamma} l} t, \quad X_{i}(t)=Q_{i}+t \frac{P_{i}}{\sqrt{\gamma} l}
$$

Эти формулы устанавливают связь между координатами системы покоя, в которой наблюдатель измеряет полную энергию струны $P_{0}$ и время $X_{0}$, а также изменение координат “центра масс" струны $X_{i}$ со временем $X_{0}$

$$
X_{i}\left(X_{0}\right)=Q_{i}+X_{0} \frac{P_{i}}{P_{0}}
$$

с координатами схемы Леви-Чивита $\left(t, H, \Pi_{i}, Q_{i}\right)$, в которой $t$ есть время сопутствующей "центру масс" системы отсчета, $\Pi_{i}$ и $Q_{i}$ оказываются циклическими переменными.

\section{5. ДИНАМИКА ЛОКАЛЬНЫХ ПЕРЕМЕННЫХ}

5.1. Репараметризационно-инвариантная редукция для открытой струны. Рассмотрим открытую струну с граничными условиями

$$
\sigma_{1}(\bar{\tau})=0, \quad \sigma_{2}(\bar{\tau})=\pi, \quad l(\bar{\tau})=\pi, \quad \xi_{\mu}^{\prime}(t, 0)=\xi_{\mu}^{\prime}(t, \pi)=0 .
$$

Фиксируя калибровку, как в (51), положим

$$
\bar{\lambda}_{1}(\tau, \sigma)=1, \quad \bar{\lambda}_{2}(\tau, \sigma)=0
$$

что не противоречит условию нормировки функции смещения $\bar{\lambda}_{1}(48)$. В результате из (50) имеем

$$
\mathcal{H}-\frac{1}{\pi} \int_{0}^{\pi} d \sigma \mathcal{H}=0, \quad \pi_{\mu} \xi^{\prime \mu}=0 .
$$

Первое равенство в (68) означает, что редуцированная плотность гамильтониана $\mathcal{H}(46)$ совпадает с самим гамильтонианом $H / \pi$. Уравнения для локальных переменных, получаемые варьированием редуцированного действия (61),

$$
\frac{\delta W^{\mathrm{R}}}{\delta \xi^{\mu}}=0 \Rightarrow \partial_{t} \pi_{\mu}-\partial_{\sigma}\left(\bar{\lambda}_{2} \pi_{\mu}\right)=\gamma \partial_{\sigma}\left(\bar{\lambda}_{1} \xi_{\mu}^{\prime}\right), \quad \frac{\delta W^{\mathrm{R}}}{\delta \pi^{\mu}}=0 \Rightarrow \gamma D_{t} \xi_{\mu}=\bar{\lambda}_{1} \pi_{\mu}
$$


при условии (67) снова приводят к линейному уравнению Даламбера (15), где теперь временной параметр $\tau$ заменяется на собственное время "центра масс" $t$,

$$
\partial_{t}^{2} \xi_{\mu}-\partial_{\sigma}^{2} \xi_{\mu}=0
$$

Обшее решение этих уравнений движения для $\xi_{\mu}$ и $\pi_{\mu}$ в классе функций $(37),(41)$ с границей (66) дается фурье-рядами (17)

$$
\begin{array}{ll}
\xi_{\mu}(t, \sigma)=\frac{1}{2 \sqrt{\pi \gamma}}\left[\psi_{\mu}\left(z_{+}\right)+\psi_{\mu}\left(z_{-}\right)\right], & \psi_{\mu}(z)=i \sum_{n \neq 0} e^{-i n z} \frac{\alpha_{n \mu}}{n}, \quad z_{ \pm}=t \pm \sigma, \\
\xi_{\mu}^{\prime}(t, \sigma)=\frac{1}{2 \sqrt{\pi \gamma}}\left[\psi_{\mu}^{\prime}\left(z_{+}\right)-\psi_{\mu}^{\prime}\left(z_{-}\right)\right], & \pi_{\mu}(t, \sigma)=\frac{1}{2} \sqrt{\frac{\gamma}{\pi}}\left[\psi_{\mu}^{\prime}\left(z_{+}\right)+\psi_{\mu}^{\prime}\left(z_{-}\right)\right] .
\end{array}
$$

Глобальные координаты $Q_{\mu}$ и импульсы $P_{\mu}$ определены редуцированной динамикой “центра масс" (63)-(65), а масса струны $M$ определяется глобальной связью (46),

$$
P_{\mu}^{2}=M^{2}=2 \pi \gamma H=2 \pi \gamma \int_{0}^{\pi} d \sigma \mathcal{H}
$$

Подстановка $\xi_{\mu}$ и $\pi_{\mu}$ из (71) в (43) приводит к представлению плотности гамильтониана $\mathcal{H}$ через функции $\psi_{\mu}^{\prime}\left(z_{ \pm}\right)$,

$$
\mathcal{H}=-\frac{1}{4 \pi}\left[\psi_{\mu}^{\prime 2}\left(z_{+}\right)+\psi_{\mu}^{\prime 2}\left(z_{-}\right)\right]
$$

а из (72) получаем для квадрата массы струны выражение, совпадаюшее с (24).

Из локальных связей (68), записанных в терминах функций $\psi_{\mu}^{\prime}\left(z_{ \pm}\right)$, через которые выражаются $\xi_{\mu}^{\prime}$ и $\pi^{\mu}$ в $(71)$, имеем

$$
\begin{gathered}
\xi_{\mu}^{\prime} \pi^{\mu}=\frac{1}{4 \pi}\left[\psi_{\mu}^{\prime 2}\left(z_{+}\right)-\psi_{\mu}^{\prime 2}\left(z_{-}\right)\right]=0 \Rightarrow \psi_{\mu}^{\prime 2}(\tau+\sigma)=\psi_{\mu}^{\prime 2}(\tau-\sigma), \\
\psi_{\mu}^{\prime 2}\left(z_{+}\right)+\psi_{\mu}^{\prime 2}\left(z_{-}\right)=\frac{1}{\pi} \int_{0}^{\pi} d \sigma\left[\psi_{\mu}^{\prime 2}(\tau+\sigma)+\psi_{\mu}^{\prime 2}(\tau-\sigma)\right],
\end{gathered}
$$

откуда следует, что квадрат модуля вектора $\psi_{\mu}^{\prime}(z)$ есть величина постоянная, т.е. $\psi_{\mu}^{\prime 2}(z)=$ const. Из формулы (72) вытекает, что эта постоянная задается величиной

$$
\psi_{\mu}^{\prime 2}(z)=-\frac{M^{2}}{\pi \gamma}
$$

Таким образом, функции $\psi_{\mu}(z)$ подчиняются условию

$$
P_{\mu}^{2}+\pi \gamma \psi_{\mu}^{\prime 2}\left(z_{ \pm}\right)=0 \quad\left(P_{\mu}^{2}=M^{2}\right),
$$

в отличие от связи (20) в стандартном подходе

$$
\left(P_{\mu}+\sqrt{\pi \gamma} \psi_{\mu}^{\prime}\right)^{2}=0
$$


Уравнение (76) содержит интерференционный член глобальной и локальных степеней свободы $\sqrt{\pi \gamma} \psi_{\mu}^{\prime}(z) P^{\mu}$, который нарушает релятивистскую инвариантность локальных возбуждений струны, формируюших ее массу и спин.

Связи (75) и (76) совпадают только для калибровки Рорлиха [23] в стандартном подходе, когда вместо произвольного времениподобного вектора $n_{\mu}$ в $(51)$ выбирается полньй импульс струны $P_{\mu}$.

Из условия (75) следует, что вектор $\psi_{\mu}^{\prime}$ - пространственноподобный, постоянного модуля, а вектор $P_{\mu}$ - времениподобный. При $M=0$ оба этих вектора становятся изотропными (светоподобными). Связь (75) в терминах коэффициентов Фурье (71) принимает следуюший вид:

$$
\psi_{\mu}^{\prime 2}(z)=\sum_{n, m \neq 0} \alpha_{n \mu} \alpha_{m}^{\mu} e^{-i(n+m) z}=\sum_{N} e^{-i N z} \sum_{n \neq 0, N} \alpha_{n \mu} \alpha_{N-n}^{\mu}=-\frac{M^{2}}{\pi \gamma} .
$$

На уровне величин $\bar{L}_{n}$, введенных в стандартном формализме (23), репараметризационно-инвариантная связь $(77)$ означает, что ее нулевая гармоника $\bar{L}_{0}$, определяюшая массу струны,

$$
\bar{L}_{0}=-\frac{1}{2} \sum_{n \neq 0} \alpha_{n \mu} \alpha_{-n}^{\mu}=-2 \pi \gamma M^{2},
$$

осталась той же самой, как и в случае с фиксацией калибровки, в то время как ненулевые гармоники $\bar{L}_{N \neq 0}$ связи $(77)$

$$
\bar{L}_{N \neq 0}=-\frac{1}{2} \sum_{n \neq 0, N} \alpha_{n \mu} \alpha_{N-n}^{\mu}=0, \quad \bar{L}_{-N}=\bar{L}_{N}^{*}
$$

существенно отличаются от связей с фиксацией калибровки (20) тем, что они не содержат глобального импульса струны $P_{\mu}=\sqrt{\pi \gamma} \alpha_{0 \mu}$ (суммы в (78) и (79) не содержат $\left.\alpha_{0 \mu}\right)$. Другими словами, нулевые моды $\alpha_{0 \mu}$, т.е. импульсы "центра масс" $P_{\mu}$, входят в нашем подходе только в глобальную (энергетическую) связь (72) $P_{\mu}^{2}=M^{2}$, где масса струны задается суммой вкладов внутренних возбуждений струны в (78), в то время как (79) связывает только коэффициенты Фурье этих внутренних возбуждений $\alpha_{n, \mu}$ при $n \neq 0$.

Как уже отмечалось выше, связи (75), а следовательно, и (78), (79) возникают и в стандартном подходе (см. (76)), если воспользоваться калибровочным условием Рорлиха [23], в котором вместо произвольного вектора $n_{\mu}$ в $(51)$ используется полньй импульс струны $P_{\mu}[14]$,

$$
P_{\mu} x^{\mu}(\tau, \sigma)=\frac{P_{\mu}^{2}}{\pi \gamma} \tau+P_{\mu} Q^{\mu}, \quad P_{\mu} x^{\prime \mu}=0 .
$$

Отсюда с учетом вида решения для $x_{\mu}(\tau, \sigma)$ в (16) имеем

$$
P_{\mu} \psi_{\mu}\left(z_{ \pm}\right)=0
$$

и, следовательно, условия (75) и (76) становятся эквивалентными. 
Однако отличие калибровочного подхода от репараметризационно-инвариантного связано с определением временно́й координаты и соответствуюшего генератора эволюции (т.е. гамильтониана). В репараметризационно-инвариантном подходе собственное время $t$ возникает из динамической переменной “центра масс" струны $X_{0}$ в результате канонического преобразования Леви-Чивита и явного решения глобальной связи (cм. $(55))$

$$
X_{0}(t)=\frac{P_{0}}{\pi \sqrt{\gamma}} t .
$$

Гамильтониан эволюции относительно собственного времени не равен нулю в методе инвариантной редукции (61). В то же время в калибровочном подходе роль параметра эволюции играет безразмерное координатное время $\tau$, которое на классических решениях задает эволюцию всех переменных, в частности

$$
X_{0}(\tau)=\frac{P_{0}}{\pi \gamma} \tau
$$

однако с нулевым дираковским гамильтонианом.

В нашем подходе калибровка Рорлиха (81) означает, что

$$
P_{0} \psi_{0}(z)=0 \Rightarrow P_{0} \alpha_{n, 0}=0, \quad n \neq 0,
$$

и при $P_{0}=M \neq 0$ все временны́е фурье-компоненты $\alpha_{n, 0}=0$ для $n \neq 0$ (это эквивалентно кулоновской калибровке $A_{0}=0$ в электродинамике). Окончательно локальные связи определятся выражениями $\bar{L}_{N \neq 0}=0$, т.к.

$$
\psi_{i}^{\prime 2}(z)=\frac{M^{2}}{\pi \gamma} \Rightarrow M^{2}=\pi \gamma \sum_{n \neq 0} \alpha_{n, i} \alpha_{n, i}^{*}, \quad \bar{L}_{N}=\sum_{n \neq 0, N} \alpha_{n, i} \alpha_{N-n, i}=0
$$

которые вместе с $\bar{L}_{0}$ образуют замкнутую алгебру скобок Пуассона

$$
\left\{\bar{L}_{N}, \bar{L}_{M}\right\}=-i(N-M) \bar{L}_{N+M} .
$$

5.2. Квантовая теория. Рассмотрим на операторном уровне предложенную выше гамильтонову динамику открытой релятивистской струны с возникающими в ней связями первого рода (50), (79) и второго рода (82), которая, как было отмечено, соответствует подходу Рорлиха. Заметим, что условие (82) позволяет исключить состояния с отрицательной нормой, поскольку постулируемые коммутационные соотношения

$$
\left[\alpha_{m, \mu}, \alpha_{n, \mu}^{+}\right]=-m \eta_{\mu, \nu} \delta_{m, n}, \quad n, m \neq 0, \quad \eta_{00}=\eta_{i i}=1,
$$

приводят для временны́х компонент $\alpha_{n, 0}^{+}$при их действии на вакуум к векторам состояния с отрицательной нормой. Имея теперь только пространственные компоненты операторов рождения и уничтожения $a_{n, i}^{+}=\alpha_{-n, i} \sqrt{n}, a_{n, i}=\alpha_{n, i} \sqrt{n}$, можно в системе “центра масс" построить нормированный вектор состояния [23]

$$
\left|\Phi_{\nu}\right\rangle_{\text {цM }}=\prod_{n=1}^{\infty} \frac{\left(a_{n x}^{+}\right)^{\nu_{n x}}}{\sqrt{\nu_{n x} !}} \frac{\left(a_{n y}^{+}\right)^{\nu_{n y}}}{\sqrt{\nu_{n y} !}} \frac{\left(a_{n z}^{+}\right)^{\nu_{n z}}}{\sqrt{\nu_{n z} !}}|0\rangle
$$


где числа $\nu_{n x}, \nu_{n y}, \nu_{n z}$ принимают неотрицательные целые значения. Этот вектор состояния автоматически удовлетворяет условию

$$
a_{n, 0}\left|\Phi_{\nu}\right\rangle_{\text {LM }}=0, \quad n>0
$$

а также связям (78), (79), налагаемым на него только для $N>0$,

$$
\bar{L}_{N}\left|\boldsymbol{\Phi}_{\nu}\right\rangle_{\text {цМ }}=0, \quad N>0, \quad M_{\nu}^{2}=\pi \gamma\left\langle\boldsymbol{\Phi}_{\nu} \sum_{n \neq 0} \alpha_{n, i} \alpha_{n, i}^{+} \mid \boldsymbol{\Phi}_{\nu}\right\rangle,
$$

поскольку оператор $\bar{L}_{N}$ для $N>0$ может быть всегда представлен в нормальной форме

$$
\bar{L}_{N>0}=\sum_{n>0}^{\infty} \alpha_{n, i}^{+} \alpha_{N+n, i}+\frac{1}{2} \sum_{n=1}^{N-1} \alpha_{n, i} \alpha_{N-n, i} .
$$

Оператор $\bar{L}_{N}$ для $N<0$ согласно (79) является эрмитово сопряженным к $\bar{L}_{N}, \bar{L}_{-N}^{+}=$ $\bar{L}_{N}$, и поэтому вместо первого равенства (88) для него будет выполняться равенство

$$
\left\langle\boldsymbol{\Phi}_{\nu}\right| \bar{L}_{N}^{+}=0
$$

Таким образом, нам необходимо рассмотреть алгебру операторов $\alpha_{n, 0}($ для $n>0)$ и $\bar{L}_{N}$ (для $N>0$ ), обрашающих в нуль вектор состояния аналогично квантовой электродинамике, где условие Лоренца $\partial A_{\mu}^{(-)} / \partial x_{\mu}|\boldsymbol{\Phi}\rangle=0$ содержит только аннигилирующую часть вектор-потенциала. Эти операторы согласно классификации Дирака [8] дают связи первого рода, образуя замкнутую алгебру (только для $n, N>0$ ),

$$
\left[\alpha_{n, 0}, \alpha_{n, 0}\right]=0, \quad\left[\alpha_{n, 0}, \bar{L}_{N}\right]=n \alpha_{N+n, 0}, \quad\left[\bar{L}_{N}, \bar{L}_{M}\right]=(N-M) \bar{L}_{N+M}
$$

Поэтому условия (87) и (88) оказываются самосогласованными в противоположность обшепринятому рассмотрению, когда при произвольных $N, M$ последний коммутатор в (89) содержит $c$-числовой член,

$$
\left[\bar{L}_{N}, \bar{L}_{M}\right]=(N-M) \bar{L}_{N+M}+\frac{D}{12} N\left(N^{2}-1\right) \delta_{N,-M}
$$

а второй коммутатор в (89) при $n+N<0$ содержит в правой части оператор рождения $\alpha_{-N-n, 0}^{+}$.

Итак, на операторном уровне связи разрешаются в слабом смысле, и только "аннигилирующая" часть операторов налагается на вектор состояния.

В квантовой теории можно ввести полный набор собственных (осцилляторных) функций, удовлетворяющих уравнению

$$
H\left[\pi_{i}, \xi_{i}\right]\langle\xi \mid \nu\rangle=\frac{M_{\nu}^{2}}{2 \pi \gamma}\langle\xi \mid \nu\rangle,
$$

где

$$
\langle\xi \mid \nu\rangle=\left\langle\xi \mid \Phi_{\nu}\right\rangle, \quad \sum_{\nu}\left\langle\xi_{1} \mid \nu\right\rangle\left\langle\nu \mid \xi_{2}\right\rangle=\prod_{\sigma} \delta^{3}\left(\xi_{1}-\xi_{2}\right) .
$$




\section{6. ПРИЧИННЫЕ ФУНКЦИИ ГРИНА}

Мы можем построить причинные функции Грина для релятивистской струны по аналогии со случаем релятивистской частишы с массой $M_{\nu}[22]$,

$$
\begin{aligned}
G_{\mathrm{c}}(X \mid \nu) & =G_{+}(X \mid \nu) \theta\left(X_{0}\right)+G_{-}(X \mid \nu) \theta\left(-X_{0}\right)= \\
& =i \int \frac{d^{4} P}{(2 \pi)^{4}} \exp (-i P X) \frac{1}{P^{2}-M_{\nu}^{2}-i \epsilon},
\end{aligned}
$$

где $G_{+}(X \mid \nu)=G_{-}(-X \mid \nu)$ - коммутативная функция Грина [22]

$$
G_{+}(X \mid \nu)=\int \frac{d^{4} P}{(2 \pi)^{3}} \exp (-i P X) \delta\left(P^{2}-M_{\nu}^{2}\right) \theta\left(P_{0}\right) .
$$

Последняя может быть представлена в форме функционального интеграла по полному фазовому пространству $P_{\mu}, X_{\mu}[15]$

$$
\begin{aligned}
G_{+}(X \mid \nu)= & \int_{X\left(\tau_{1}\right)=0}^{X\left(\tau_{2}\right)=X} \frac{d N_{0}\left(\tau_{2}\right) d^{4} P\left(\tau_{2}\right)}{(2 \pi)^{3}} \times \\
& \times \prod_{\tau_{1} \leqslant \tau<\tau_{2}}\left\{d \bar{N}_{0}(\tau) \prod_{\mu}\left(\frac{d P_{\mu}(\tau) d X_{\mu}(\tau)}{2 \pi}\right)\right\} \exp \left(i W\left[P, X, N_{0}, M_{\nu}\right]\right),
\end{aligned}
$$

где $\bar{N}_{0}=N_{0} / 2 \pi \delta(0), \delta(0)=\int d N_{0}$ есть бесконечный объем группы репараметризации координатного времени и

$$
W\left[P, X, N_{0}, M_{\nu}\right]=\int_{\tau_{1}}^{\tau_{2}} d \tau\left[-P_{\mu} \dot{X}^{\mu}-\frac{N_{0}}{2 \pi \gamma}\left(-P_{\mu}^{2}+M_{\nu}^{2}\right)\right]
$$

есть расширенное действие для релятивистской механики [3], [15] в гамильтоновой форме.

Причинную функцию Грина для релятивистской струны (типа Венециано) естественно определить как спектральное разложение по физическим векторам состояния $\left|\boldsymbol{\Phi}_{\nu}\right\rangle=$ $|\nu\rangle$,

$$
\begin{aligned}
G_{\mathrm{c}}\left(X \mid \xi_{1}, \xi_{2}\right) & =G_{+}\left(X \mid \xi_{1}, \xi_{2}\right) \theta\left(X_{0}\right)+G_{-}\left(X \mid \xi_{1}, \xi_{2}\right) \theta\left(-X_{0}\right)= \\
& =i \int \frac{d^{4} P}{(2 \pi)^{4}} \exp (-i P X) \sum_{\nu} \frac{\left\langle\xi_{1} \mid \nu\right\rangle\left\langle\nu \mid \xi_{2}\right\rangle}{P^{2}-M_{\nu}^{2}-i \epsilon},
\end{aligned}
$$

где $\langle\xi \mid \nu\rangle$ - решения уравнения (90).

Коммутативные функции Грина для релятивистской струны $G_{+}\left(X \mid \xi_{1}, \xi_{2}\right)$ могут быть переписаны в форме функционального интеграла Фаддеева-Попова [25] в калибровке (51) (где $\left.n_{\mu}=(1,0,0,0)\right)$

$$
\begin{aligned}
G_{+}\left(X \mid \xi_{2}, \xi_{1}\right)= & \int_{X\left(\tau_{1}\right)=0}^{X\left(\tau_{2}\right)=X} \frac{d N_{0}\left(\tau_{2}\right) d^{4} P\left(\tau_{2}\right)}{(2 \pi)^{3}} \times \\
& \times \prod_{\tau_{1} \leqslant \tau<\tau_{2}}\left\{d \bar{N}_{0}(\tau) \prod_{\mu}\left(\frac{d P_{\mu}(\tau) d X_{\mu}(\tau)}{2 \pi}\right)\right\} F_{+}\left(\xi_{2}, \xi_{1}\right) .
\end{aligned}
$$


Используя представление спектрального разложения

$$
F_{+}\left(\xi_{2}, \xi_{1}\right)=\sum_{\nu}\left\langle\xi_{2} \mid \nu\right\rangle \exp \left\{i W\left[P, X, N_{0}, M_{\nu}\right]\right\}\left\langle\nu \mid \xi_{1}\right\rangle
$$

функцию $F_{+}$в $(96)$ можно записать в виде функционального интеграла

$$
F_{+}\left(\xi_{2}, \xi_{1}\right)=\int_{\xi_{1}}^{\xi_{2}} D(\xi, \pi) \Delta_{\Phi \Pi} \exp \left\{i W_{\Phi \Pi}\right\}
$$

где $W\left[P, X, N_{0}, M_{\nu}\right]$ есть действие $(94)$,

$$
W_{\Phi \Pi}=\int_{0}^{\tau\left(X_{0}\right)} d \tau\left[-\left(\int_{0}^{\pi} d \sigma \pi_{\mu} \dot{\xi}^{\mu}\right)-P_{\mu} \dot{X}^{\mu}-N_{0}\left(-\frac{P^{2}}{2 \pi \gamma}+H^{\mathrm{R}}\right)\right]
$$

есть действие на поверхности связи (50),

$$
D(\xi, \pi)=\prod_{\tau, \sigma} \prod_{\mu} \frac{d \xi_{\mu} d \pi_{\mu}}{2 \pi}
$$

и

$$
\Delta_{\Phi \Pi}=\prod_{\tau, \sigma} \delta\left(\phi_{1}\right) \delta\left(\pi_{0}\right) \delta\left(\phi_{2}\right) \delta\left(\xi_{0}\right) \operatorname{det} B^{-1}, \quad \operatorname{det} B=\operatorname{det}\left\{\phi_{1}, \phi_{2}, \pi_{0}, \xi_{0}\right\}
$$

есть детерминант Фаддеева-Попова [7].

\section{7. ЗАКЛЮЧЕНИЕ}

Репараметризационно-инвариантная редукция в гамильтоновом описании динамики релятивистской струны проводилась путем введения собственного времени "центра масс" струны и соответствующего ему ненулевого гамильтониана эволюции, определение которых возможно в рамках схемы Арновита-Дезера-Мизнера и связанной с ней группой диффеоморфизмов гамильтонова описания. Для репараметризационно-инвариантного описания необходимым являлось отделение в расширенном действии координаты "центра масс" струны в противоположность стандартному подходу, где отождествление этих координат происходит уже в самих решениях уравнений движения.

Результатом отделения переменных “центра масс" на уровне действия явилось то, что инвариантные локальные связи не содержали полный момент струны, в противоположность связям в стандартном калибровочном подходе. Поэтому возникает вопрос: когда необходимо отделять переменные "центра масс" струны - до вариации функции действия, как сделано в этой статье, или после, в уравнениях движения? Релятивистская инвариантность диктует первый способ, поскольку наблюдатель, находящийся в системе "центра масс", не может измерять полный импульс струны.

Нами показано, что сушествует калибровка Рорлиха, для которой эти два подхода ведут к одному и тому же результату. Репараметризационно-инвариантное описание 
свободно от трудностей подхода Рорлиха для введения взаимодействия. Этому вопросу будет посвящена отдельная работа.

Локальные связи не зависят от глобальных переменных ("центра масс") и отличаются от стандартных тем, что в квантовом случае ведут к новой алгебре для операторов, которая не содержит трудностей, характерных для алгебры Вирасоро (включая ограничение $D=26$ ). Причинная функция Грина релятивистской струны сконструирована в форме интеграла Фаддеева-Попова.

\section{ПРИЛОЖЕНИЕ}

Кинеметрические преобразования дифференциалов

$$
\tilde{\tau}=\dot{f}_{1}(\tau) d \tau, \quad d \tilde{\sigma}=\dot{f}_{2}(\tau, \sigma) d \tau+f_{2}^{\prime}(\tau, \sigma) d \sigma
$$

соответствуют преобразованиям координат струны

$$
\begin{gathered}
x_{\mu}(\tau, \sigma)=\tilde{x}_{\mu}(\tilde{\tau}, \tilde{\sigma}), \quad x_{\mu}^{\prime}(\tau, \sigma)=\tilde{x}_{\mu}^{\prime}(\tilde{\tau}, \tilde{\sigma}) f_{2}^{\prime}(\tau, \sigma), \\
\dot{x}_{\mu}(\tau, \sigma)=\dot{\tilde{x}}_{\mu}(\tilde{\tau}, \tilde{\sigma}) \dot{f}_{1}(\tau)+\tilde{x}_{\mu}^{\prime}(\tilde{\tau}, \tilde{\sigma}) \dot{f}_{2}(\tau, \sigma) .
\end{gathered}
$$

Из этих соотношений с учетом уравнений (7) можно вывести трансформационные законы для $\lambda_{1}$ и $\lambda_{2}$

$$
\begin{aligned}
& \lambda_{1}(\tau, \sigma)=\frac{\sqrt{\left(\dot{x} x^{\prime}\right)^{2}-\dot{x}^{2} x^{\prime 2}}}{x^{\prime 2}(\tau, \sigma)}=\frac{\sqrt{\left(\dot{\tilde{x}} \tilde{x}^{\prime}\right)^{2}-\dot{\tilde{x}}^{2} \tilde{x}^{\prime 2}}}{\tilde{x}^{\prime 2}(\tilde{\tau}, \tilde{\sigma})} \frac{\dot{f}_{1}}{f_{2}^{\prime}}=\tilde{\lambda}_{1} \frac{\dot{f}_{1}(\tau)}{f_{2}^{\prime}(\tau, \sigma)}, \\
& \lambda_{2}(\tau, \sigma)=\frac{\dot{x} x^{\prime}}{x^{\prime 2}}=\frac{\left(\dot{\tilde{x}} \tilde{x}^{\prime}\right) \dot{f}_{1} f_{2}^{\prime}+\tilde{x}^{\prime 2} \dot{f}_{2} f_{2}^{\prime}}{\tilde{x}^{\prime 2} f_{2}^{\prime 2}}=\tilde{\lambda}_{2} \frac{\dot{f}_{1}}{f_{2}^{\prime}}+\frac{\dot{f}_{2}}{f_{2}^{\prime}} .
\end{aligned}
$$

Из этих трансформационных законов и преобразований конформного фактора $\Omega(\tau, \sigma)=$ $f_{2}^{\prime}(\tau, \sigma) \widetilde{\Omega}(\tilde{\tau}, \tilde{\sigma})$ следует кинеметрическая инвариантность интервала (3) относительно преобразований (6). Ковариантная производная (5) преобразуется при учете (6) как

$$
D_{\tau} x_{\mu}=\dot{x}_{\mu}-\lambda_{2} x_{\mu}^{\prime}=\dot{f}_{1}(\tau)\left(\dot{\tilde{x}}_{\mu}-\tilde{\lambda}_{2} \tilde{x}_{\mu}^{\prime}\right)=\dot{f}_{1}(\tau) D_{\tilde{\tau}} \tilde{x}_{\mu} .
$$

Благодарности. Мы благодарны за интерес к работе и критические обсуждения А. В. Ефремову, В. Г. Кадьшевскому, Э. А. Кураеву, В. В. Нестеренко, А. И. Пашневу.

\section{Список литературы}

[1] R. Arnovitt, S. Deser, C. W. Misner. Phys. Rev. 1959. V. 116. P. 1322; 1961. V. 122. P. 997.

[2] C. Isham, K. Kuchar. Ann. Phys. (NY). 1985. V. 164. P. 288, 316; J. B. Hartle, K. Kuchar. Phys. Rev. D. 1986. V. 34. P. 2323; P. Hajicek, K. Kuchar. Phys. Rev. D. 1990. V. 41. P. 1091; P. Hajicek. Nucl. Phys. Proc. Suppl. 1997. V. 57. P. 115.

[3] L. Gyngazov, M. Pawlowski, V. Pervushin, V. Smirichinski. Gen. Relat. Gravit. 1998. V. 30. P. 1749; M. Pawlowski, V. Papoyan, V. Pervushin, V. Smirichinski. Phys. Lett. B. 1998. V. 444. P. 293. 
[4] G. Fulop, D. Gitman, I. Tyutin. Int. J. Theor. Phys. 1999. V. 38. P. 1941.

[5] V. Pervushin, V. Smirichinski. J. Phys. A. 1999. V. 32. P. 6191.

[6] П. А. М. Дирак. Лекции по квантовой механике. М.: Мир, 1968.

[7] A. J. Hanson, T. Regge, C. Teitelboim. Constrained Hamiltonian Systems. Rome: Accademia Nazionale dei Lincei, 1976.

[8] Д. М. Гитман, И. В. Тютин. Каноническое квантование полей со связями. М: Наука, 1986.

[9] Ю. С. Владимиров. Системы отсчета в теории гравитации. М.: Энергоатомиздат, 1982.

[10] P. A. M. Dirac. Proc. Roy. Soc. A. 1958. V. 246. P. 333; Phys. Rev. 1959. V. 114. P. 924.

[11] R. Arnovitt, S. Deser, C. W. Misner. Phys. Rev. 1960. V. 117. P. 1525.

[12] А. Л. Зельманов. ДАН СССР. 1976. Т. 227. С. 78.

[13] М. Грин, Дж. Швари, Э. Виттен. Теория суперструн. М.: Мир, 1990.

[14] Б. М. Барбашов, В. В. Нестеренко. Модель релятивистской струны в физике адронов. М.: Энергоатомиздат, 1987.

[15] M. Pawlowski, V. Pervushin. Parametrization-invariant path integral in GR and "Big Bang" of quantum universe. hep-th/0006116; Int. J. Mod. Phys. (to be published). 2001.

[16] T. Levi-Civita. Prace Mat. -Fiz. 1906. V. 17. P. 1.

[17] S. Shanmugadhasan. J. Math. Phys. 1973. V. 14. P. 677.

[18] L. Brink, P. Vecchia, P. Howe. Phys. Lett. B. 1976. V. 65. P. 471; A. M. Polyakov. Phys. Lett. B. 1981. V. 103. P. 207.

[19] J. W. York (Jr.). Phys. Rev. Lett. 1971. V. 26. P. 1658; K. Kuchar. J. Math. Phys. 1971. V. 13. P. 768.

[20] Л. Бринк, М. Энно. Принципы теории струн. М.: Мир, 1991.

[21] P. Goddard, J. Goldstone, C. Rebbi, C. Thorn. Nucl. Phys. B. 1973. V. 56. P. 109.

[22] C. Швебер. Введение в релятивистскую квантовую теорию поля. М.: ИЛ, 1963.

[23] F. Röhrlich. Nucl. Phys. B. 1976. V. 112. P. 842.

[24] S. Gogilidze, A. Khvedelidze, V. Pervushin. J. Math. Phys. 1996. V. 37. P. 1760; Phys. Rev. D. 1996. V. 53. P. 2160.

[25] L. Faddeev, V. Popov. Phys. Lett. B. 1976. V. 25. P. 29.

Поступила в редакцию $8 . \mathrm{X.2000} \mathrm{г.,}$ после доработки 28.XII.2000 г. 